\title{
Empowering humanitarian medical development using grid technology
}

J. Gonzales ${ }^{1}$, S. Pomel ${ }^{1}$, V. Breton ${ }^{1}$, B. Clot $^{2}$, JL Gutknecht ${ }^{3}$, B. Irthum ${ }^{4}$, Y. Legré $^{1+5}$.

${ }^{1}$ Laboratoire de Physique Corpusculaire, CNRS-IN2P3, Campus des Cézeaux, 63177 Aubière

Cedex, France

${ }^{2}$ La Chaîne de l’Espoir, Hôpital Broussais, 96, rue Didot, 75014 Paris, France

${ }^{3}$ Clinique Saint-Joseph, 9 rue Ambert 63000 Clermont-Ferrand, France

${ }^{4}$ CHU Gabriel Montpied, 58 rue Montalembert 63000 Clermont Ferrand, France

${ }^{5}$ Université d’Auvergne - IUT, Campus des Cezeaux, 63177 Aubière Cedex, France

\section{Corresponding author :}

Vincent Breton

Laboratoire de Physique Corpusculaire

Campus des Cézeaux, 63177 Aubière Cedex, France

Tel: +33473407219

Fax: +33473264598

Email: breton@clermont.in2p3.fr

\section{Abstract}

Background : The training of local clinicians is the best way to raise the standard of medical knowledge in developing countries. This requires transferring skills, techniques and resources.

Objectives : Grid technology opens new perspectives for preparation and follow-up of medical missions in developing countries as well as support to local medical centres in terms of teleconsulting, telediagnosis, patient follow-up and e-learning.

Methods : to meet requirements of a development project of the French NPO Chain of Hope in China, we propose to deploy a grid-based federation of databases

First results and conclusions : A first protocol was established for describing the patients pathologies and their pre- and post-surgery states through a web interface in a languageindependent way. This protocol was evaluated by French and chinese clinicians during medical missions in the fall 2003. The first sets of medical patients recorded in the databases will be used to evaluate grid implementation of services. 


\section{Introduction}

The training of local clinicians is the best way to raise the standard of medical knowledge in developing countries. This requires transferring skills, techniques and resources. Medical missions by European clinicians allow such transfers through the treatment of patients and training of local clinicians. Because medical missions by their very nature are time-limited and sporadic, more enduring links can be built by the renovation and construction of regional hospitals and training centres.

Chain of Hope is a humanitarian organization operating surgical missions in developing countries to train local surgeons as well as treat children. As these missions are limited in time, key issues for their success are the identification of patients needing surgery before the mission and their post-surgery follow-up after the mission. Another issue is related to the transfer of skills and techniques which should not be limited to the mission durations. Communication means are needed to keep exchange of information between European and local clinicians for remote consulting.

\section{The grid impact}

Today, a variety of telemedecine services are made available to clinicians taking advantage of the development of Internet. However, web gives access to information but the users is in charge of every steps of information exchange. At the provider end, only data made available on a server are accessible. At the customer end, finding data, storing and analyzing them stays in the hands of the user. As a result, most of the existing telemedecine services require the clinician to have several working sessions in front of his computer. For instance, telediagnosis requires often to download patient information from a server and exchanges of mails between the local physician and the clinician. Videoconferences allow interactive dialog between clinicians but interactive access to the data is not available during these sessions.

Grid technology opens perspectives to empower existing telemedicine services in terms of interactivity and access to data. Today the GRID paradigm offers computing resources and high bandwidth capacities to the users. The interconnection of computers using the GRID middleware allows the user to consume computing time and retrieve information without having to choose which machine he/she wants to connect to [1]. Several projects across Europe deploy pilot biomedical applications on test beds such as DataGrid [2]. 
As grid technology allows mutualisation of geographically distributed resources and transparent access to data, they allow European and local physicians to share patient data without having to move them around on internet networks. Existing telemedicine services for second diagnosis require physicians to transfer patient data to a repository where the clinician can download them. If information necessary for the second diagnosis is missing, the clinician must send a mail to the physician requesting additional information to be transferred to the repository. Such procedure is inefficient as it requires several actions of the clinician that must take place in different working sessions.

Grid allows the federation of patient databases located in different medical centres. A model for such federation is provided by the Mammogrid [3] project in Europe and BIRN project [4] in the United States.

These databases can be accessed from any grid site provided access rights are given. For instance, a physician in a developing country can grant to a clinician a complete read access to a patient file for telediagnosis. To do so, the physician does not have to send any information on the network and the clinician does not have to download it. The information is available to the clinician when he is available. Another example is related to the post-treatment follow-up of patients following surgical missions.

\section{First project}

The potential grid impact is being assessed on one project of the French Non Profit Organization "Chain of Hope” for medical development in China.

\section{La Chaîne de l'Espoir (Chain of Hope), non profit organization}

La Chaîne de l'Espoir (CdE), a non-profit humanitarian organization, was created in 1988 by Prof. Alain Deloche, MD, heart surgeon, to combat the geographic injustices in the availability of medical treatment and offer children from developing countries the life-saving medical and surgical care they needed.

Initially faced with numerous life or death cases, the CdE brought a large number of children from Africa, Asia and Latin America to Europe for surgery. Over 6,000 children have been operated on through the CdE and 600 children will receive surgical care in 2002 alone.

However, it was realized soon after the foundation of CdE that action was required locally, in the children's home countries, and that this action should involve local surgeons and consist of 
sustainable development rather than temporary, emergency missions. The main purpose of the $\mathrm{CdE}$ is the treating and curing of children, but equally it is the training to cure of local surgeons in developing countries. In this way the children can remain in their own country, within the security of their own culture, but receive the same standard of care that they would in Europe. In order to achieve this goal, the CdE is committed to support technology and know-how transfer programs suited to local needs and demands.

Therefore, the CdE began to operate surgical missions in Asia, Africa and Latin America; these missions included the assessment and training of local surgeons and medical staff as well as the treatment of children. In most cases, local medical practitioners and surgeons were also taken as trainees at European hospitals.

The CdE then took the obvious next step: contribute to the development of sustainable surgical units that address specific local needs and are run by well-trained local personnel with the highest-standard of equipment. With these objectives in mind, the CdE established the Maputo Heart Institute in Mozambique (June 2001) and the Phnom Penh Heart Centre in Cambodia (November 2001) which together, after only 22 months, can receive 2,000 outpatients and carry out 60 operations every month, 30 for disadvantaged children.

\section{Neuropediatric surgery in China}

Since march 2000, humanitarian missions were conducted in neurosurgical unit of the Regional Hospital of District of Chuxiong (Yunnan, PRC) by two of us (J.-L. G., B. I.) with intention of treating hydrocephalus and some other neurosurgical pathologies in children (tumours, malformations, ...). Such pathologies were previously not even treated in this hospital. These missions organised by the Chain of Hope were accomplished with the help of neurosurgical team of Professor Ding from $n^{\circ} 9$ people hospital of Shanghai.

During the seven missions that took place since March 2000, more than 200 consultations and 28 operations were realised [5].

To achieve this goal, a standard medical file was designed and translated in Chinese language. This file is subdivided in six sheets giving information concerning different steps of medical status of patient (parental operation agreement, pre-operative status, operative report, followup, complications and re-operation, anaesthetic examination).

In the elapsed time between two missions, some children previously operated were reviewed and re-operated by neurosurgeons from Chuxiong's hospital to cure a complication. Some 
others were seen and operated for hydrocephalus. Local neurosurgeons filled in the same medical files and the collected information was included in the medical files of French neurosurgeons of Chain of Hope during the next mission.

Some communication difficulties between neurosurgeons of Chuxiong's hospital speaking only Chinese language and French neurosurgeons were solved by the collaboration of neurosurgeons from $n^{\circ} 9$ people hospital of Shanghai acting as translators.

Usage of computerised medical files including clinical and radiological data shared by each medical team was identified as a relevant tool for telediagnosis of new patients medical problems, to solve some difficulties of diagnosis and to precise the indications for operation. Such usage provides also to French neurosurgeons information concerning the follow-up of patients seen by local neurosurgeons between two missions. The sharing of these computerised medical files between several medical teams (Clermont-Ferrand, Shanghai, Chuxiong) working together to achieve these missions improves knowledge and education of medical teams who less frequently treat some pathologies.

\section{First results and issues}

Both projects require the definition of a common protocol for describing the patient pathology and his pre- and post-surgery state. This description should be accessible to all physicians in their own language. We addressed this issue by defining standard sheets.

A first protocol using a web interface was set up. This solution has the advantage to be portable and can be fully developed in France before the mission. The standard sheets can be filled and read in all relevant languages : Chinese, French and English (fig. 1). Information is stored together with medical images that can be consulted through a secure interface. 


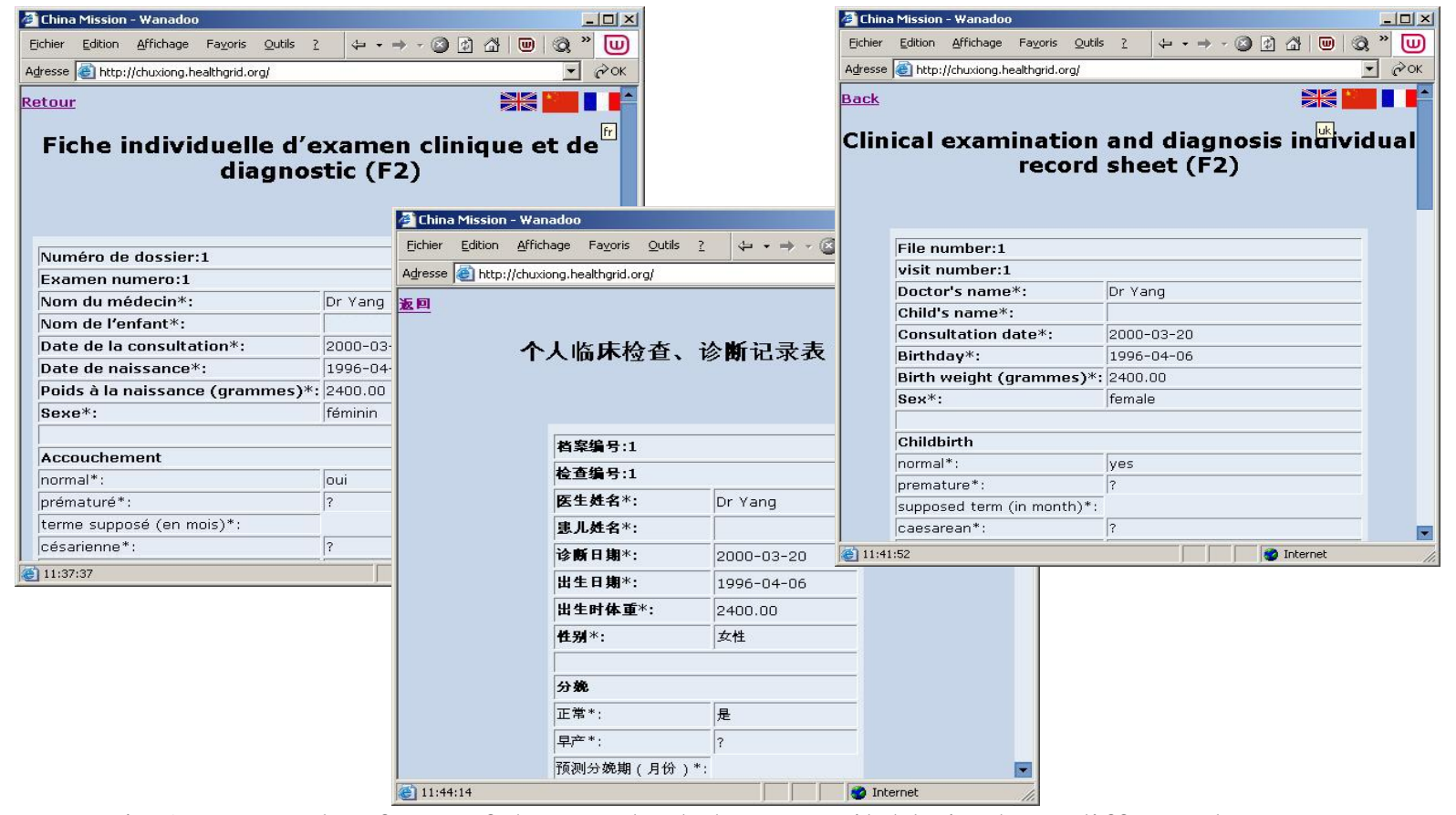

Fig.1 : Example of one of the standard sheets available in three different languages

This first protocol was tested during a mission at the Chuxiong's hospital (Yunnan, PRC) in Autumn 2003. The implementation of the web interface developed in France was used and validated on site. This first clinical use helped us to improve the sheets' description following clinician recommendations and also to assess the local network capacity necessary for the use of the web interface.

This web interface is a first step towards the use of grid technology. In the next few months we expect to install a grid node at the hospital $n^{\circ} 9$ of Shanghai and patient databases to store medical information about the humanitarian mission. The Chinese clinicians in poverty regions of western China will then fill the sheets for their patients and store it on the chinese grid node in Shanghaï. This will enable chinese clinicians to provide a second remote diagnosis and if necessary ask for additional help from their french colleagues.

\section{Conclusion}

Grid technology opens new perspectives for preparation and follow-up of medical missions in developing countries as well as support to local medical centres in terms of teleconsulting, telediagnosis, patient follow-up and e-learning. We have presented in this document an exploratory work to use grid technology to develop neuropediatric surgery in poverty regions of western China. The strategy adopted to describe the patient pathology and his pre- and 
post-surgery state through standard sheets accessible to all physicians in their own language can be generalized to any medical field. A first database of chinese patients is being built in Clermont-Ferrand and is accessible through a web interface. The project next step is the setup of a second patients database in Shanghaï on a grid node so that the two databases are federated.

\section{References}

[1] I. Foster and C. Kesselman (1999) The GRID, blueprint for a new computing infrastructure, Morgan Kaufman, San Francisco, 1999.

[2] V. Breton, R. Medina and J. Montagnat, "DataGrid, Prototype of a Biomedical Grid”, Methods MIMST, 42(2), 2003, Schattauer.

[3] Mammogrid project, EU-IST 5th framework programme, http://mammogrid.vitamib.com

[4] BIRN, The Biomedical Informatics Research Network (BIRN) sponsored by the National Institutes of Health (NIH) and the National Center for Research Resources (NCRR), http://www.nbirn.net/

[5] J. Yang, B. Peng and B. Zheng, Chinese Journal of Medical Science, June 2003, Vol 2 $\mathrm{n}^{\circ} 12,956$ 